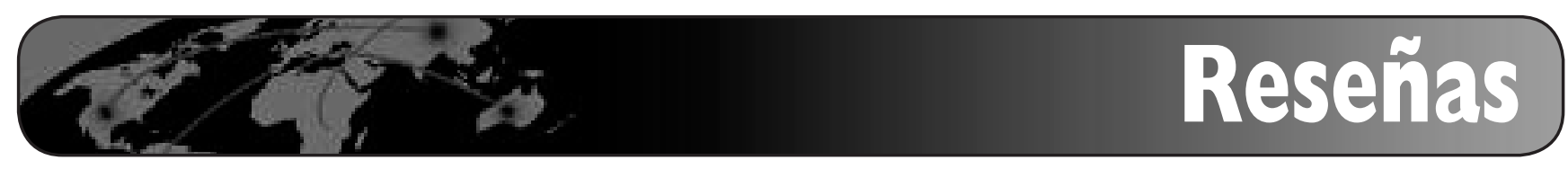

\title{
Online information conference 2007
}

\author{
Por Tomàs Baiget y Fernanda Peset
}

\begin{abstract}
Resumen: Informe de los principales conceptos y comentarios escuchados en la $31^{a}$ Online Information Conference (antiguo Online Information Meeting) de Londres, organizada por Incisive Media - VNU Exhibitions. Temas tratados: influencia Web 2.0 en las bibliotecas, comportamiento de los jóvenes como usuarios de los servicios de información, fenómeno Wikipedia, evolución de la gestión del conocimiento. También se informa del Spanish Meeting Point, un stand que sirvió de plataforma a empresas, profesionales y académicos españoles.
\end{abstract}

Palabras clave: Online information conference, Londres, Web 2.0, Spanish Meeting Point, Reseña, Informe.

Title: Online information conference 2007

Abstract: Report of the main concepts and comments heard at the 31st Online Information Conference (formerly Online Information Meeting) in London, organized by Incisive Media - VNU Exhibitions. Topics covered: Web 2.0 influence in libraries, behaviour of young people as users of information services, the Wikipedia phenomenon, the evolution of knowledge management. There is also a report of the Spanish Meeting Point, a stand that served as a platform for small businesses, professionals and academics from Spain.

Keywords: Online information conference, London, Web 2.0, Spanish Meeting Point, Conference review, Conference report.

Baiget, Tomàs; Peset, Fernanda. “Online information conference 2007”. En: El profesional de la información, 2008, v. 17, n. 5, septiembre-octubre, pp. 567-572.

DOI: $10.3145 /$ epi.2008.sep.13
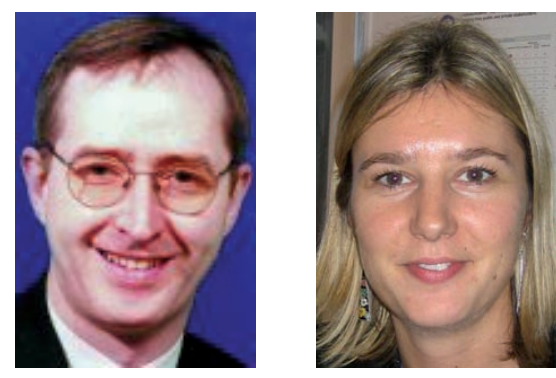

Adrian Dale, editor del Journal of Information Science, y consultor en gestión del conocimiento, se estrenó este año como presidente de la $37^{\underline{a}}$ conferencia.

Lorna Candy gestiona la Conferencia.

EL TEMA PROTAGONISTA (quizá excesivamente protagonista, habiendo tantas cosas otras de las que hablar) de la Online Information Conference (ex-Online meeting) 2007 fue la web 2.0. Ese fenómeno que cuando empezó allá por el año 2004 algunos juzgaron como algo pasajero, un juego casi, pero que ha resultado ser una revolución de gran impacto y largo alcance.
Es por ello que la key note o ponencia de apertura fue impartida por Jimmy Wales, el creador de Wikipedia, ejemplo de la Web 2.0 por antonomasia, quien aprovechó la reunión para promoverla, así como su nuevo proyecto Wikia. Wales define Wikia como una base de datos de la larga cola, en oposición a la enciclopedia colaborativa que es la Wikipedia. Puso un ejemplo sobre la diferencia entre Wikipedia y Wikia buscando información sobre muppets: la primera arroja 120 artículos sobre este tema, mientras que Wikia obtiene 17.200.

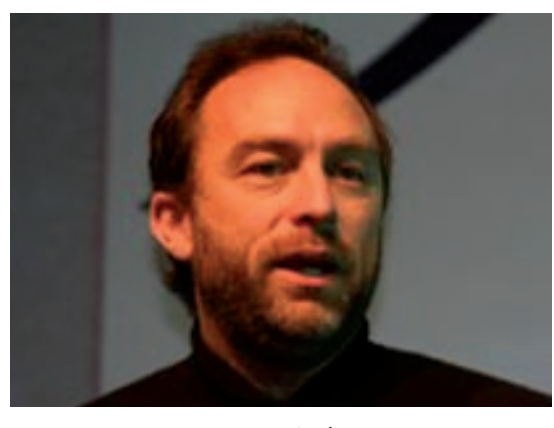

Jimmy Wales

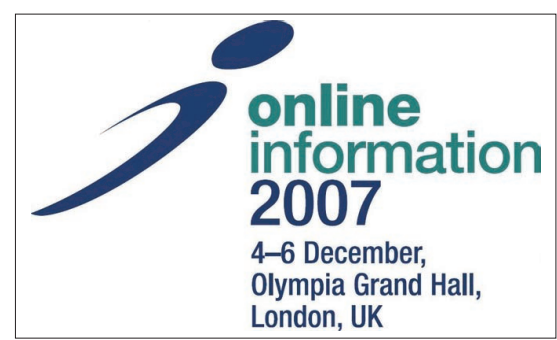

http://wikipedia.org

http://wikia.com

Wikia, fundada por Jimmy Wales y Angela Beesley en 2004, es una empresa que se autodefine como "de apoyo a la creación y el desarrollo de comunidades wiki sobre cualquier tema". En este momento (agosto de 2008) existen 6.000 comunidades en 70 idiomas, predominando las de ocio.

También habló del nuevo motor de búsqueda Wikia Search, que pretende ser "transparente, comunitario, confidencial y de calidad". El sistema, que no se financia por 


\section{La larga cola}

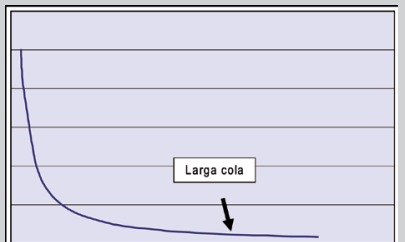

Forma informal de referirse a la parte plana de una distribución de Lévy, Pareto, Zipf, Lorenz, Bradford o Lotka (distintos enunciados de la misma ley), todas las cuales pueden aproximarse con la regla práctica del 20/80.

Chris Anderson lo puso de moda (Wired, 2004) hablando de las nuevas posibilidades de negocio en internet. Ahora existen dos tipos de mercados: uno centrado en muchas ventas ( $80 \%)$ de pocos productos (20\%) y otro, nuevo y todavía poco familiar, basado en la suma de pequeñas ventas de muchos productos, que puede igualar o superar al primero. Son el antiguo mercado de masas (pocos productos pero superventas) -la cabeza-, y los nuevos nichos de mercado, -la cola de la conocida gráfica de distribución estadística-. la publicidad, todavía da resultados bastante escasos, pero promete. Se puede ver funcionando en:

\section{http://re.search.wikia.com/}

Wales dijo que Wikipedia era como una "Cruz Roja de la información" pues es abierta, sin posibilidad de ser vendida a Google!, financiada por pequeñas donaciones, por lo que no recibe presión de ningún grupo, y tampoco depende de la publicidad. Su popularidad se extiende a todo el mundo, e incluso es utilizada en países como Irán, donde ocupa el $14^{\circ}$ puesto.

\section{Educación con Wikipedia para} escapar del hambre

Wales contó esta anécdota: con sus colegas fueron a un tugurio ocupado ilegalmente de Nueva Delhi,
India, para ver la forma en que Wikipedia les podía ayudar a compartir la información, su cultura, etc. En esos barrios marginales no había ningún servicio público, y sus habitantes corren el riesgo permanente de ser barridos de allí. Wales se ofreció para crear páginas en hindi, su idioma nativo. Ante su sorpresa, todos los padres se negaron. Como allí no llega el sistema público, ellos por su cuenta se habían creado escuelas para los niños y lo que realmente querían era simplemente usar la Wikipedia en inglés pues creen que esa es la única manera de que sus hijos en el futuro puedan salir de aquel antro. Toda la enseñanza la basan en la Wikipedia. Para ellos, su idioma local era como un "silo" que les bloquea poder salir de los barrios marginales.

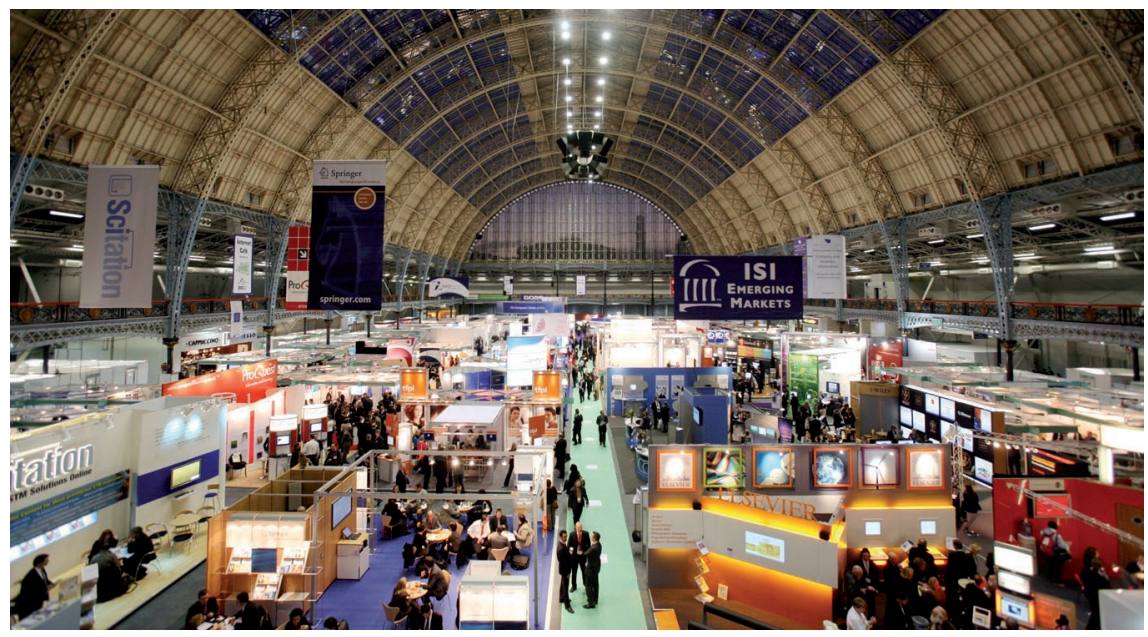

El impresionante Grand Hall del Olympia

\section{Innovación en bibliotecas}

Otra sesión en la que estuvimos fue "Biblioteca 2.0: ¿realidad o ficción?", y como era de esperar la respuesta fue una gran "realidad", ¡claro! El primer orador, Stephen Abram (vicepresidente de SirsiDynix, actual presidente de la Special Libraries Ass. y anterior presidente de la Canadian Library Ass.) se presentó como un evangelista de la web 2.0, y abundó en conceptos alrededor de "evolucionar o morir". También sacó uno de los temas repetidos en la Conferencia acerca de las supuestas proclividades de la "generación Google", los jóvenes nacidos en torno a 1990, que esencialmente no saben del mundo anterior a internet.

Neil Stewart, blogger asistente a la conferencia, comentó: "Para ser honesto, ya estoy un poco harto de que me digan que los bibliotecarios tenemos que evolucionar a causa del gran cambio de paradigma de la web 2.0 -creo que los bibliotecarios hemos estado evolucionando siempre ante la necesidad de adquirir nuevas habilidades. Y sin duda esto se aplica a todas las profesiones que utilizan información, no sólo a los bibliotecarios. Además los bibliotecarios en general, ¿es que no estamos yendo a suficiente velocidad con todo eso? En fin, que me molestan los oradores que van pontificando con tanto consejito".

David Clay, de la biblioteca de la University of Liverpool, recomendó que en las bibliotecas universitarias haya alguien experto y pueda asesorar en bibliometría al personal académico, dada la renovada importancia que va adquiriendo para evaluar a profesores e investigadores. Precisamente en este número de $E P I$ se publica una nota que comenta lo mismo ${ }^{3}$. Puso bastante énfasis en el índice $\mathrm{h}$ y en la base de datos Scopus, ya que "hace sus desarrollos en cooperación con bibliotecarios". "El mejor método para realizar la evaluación 
(research performance measurement, $R P M$ ) de un investigador es la combinación del índice $\mathrm{h}$ junto con un análisis llevado a cabo en su institución o departamento".

Jane Dysart, consultora sobre bibliotecas, de Canadá, habló sobre el uso de YouTube para promover los servicios de biblioteca. Recomendó el servicio muy firmemente como una manera de conectar con los usuarios por medios semi-formales. A pesar del aire de informalidad, la oradora subrayó la importancia de asegurar que cualquier aspecto del vídeo parezca profesional, cuidando particularmente de la calidad del sonido.

La editorial Information Today ha instituido los premios Infotubey para los mejores vídeos producidos por bibliotecas públicas. Los ganadores de 2007 se dieron a conocer durante la pasada conferencia Computers in Libraries, en Arlington, VA, en abril de 2008.

Dave Pattern, University of Huddersfield. Esta universidad está a la vanguardia de la creación de un opac verdaderamente 2.0 , y es un ejemplo de lo que puede hacerse con voluntad y suficiente imaginación. En él se han incluido canales RSS, recomendaciones de materiales de lectura similares durante la realización de búsquedas, sugerencias ortográficas al introducir los términos de búsqueda, y un sinnúmero de otras características.

\section{La conferencia en Facebook}

En Facebook se abrió una sección sobre la Online Information Conference: http://www.facebook.com/group.php?.gid=5540078274

En ella se inscribieron 450 personas. No está mal, pero la gran mayoría estuvo pasiva. http://webcat.hud.ac.uk/

Ante las cifras decrecientes de uso de las bibliotecas (se hablaba en general de un $30 \%$ entre 2005 y 2007), siempre sale la pregunta de si los bibliotecarios desaparecerán. Jimmy Wales dijo: "Todo el mundo puede contar chistes, pero los actores cómicos siguen siendo imprescindibles".

Incluso en conferencias a las que se les presupone un nivel alto de conocimientos uno se encuentra con fue la de Karen Blakeman. Explicó los servicios web 2.0, es decir, blogs, RSS, Wikis y cosas por el estilo. Creemos que cualquier persona que se gasta 1.000 euros para estar en la Online Information Conference tiene ya bastante idea de estos conceptos básicos. Sin embargo, para los que vamos allí con las eternas dificultades con el idioma, esas sesiones de repaso hasta se agradecen. presentaciones muy básicas, como

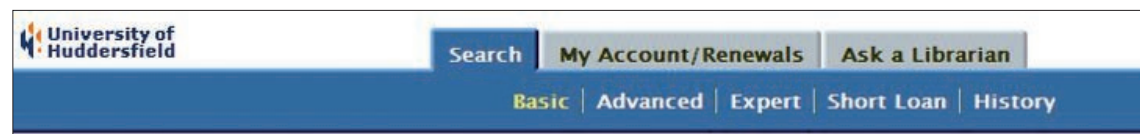

You're searching: University of Huddersfield Library

Basic Search

Search the library catalogue by selecting your desired index, input your term and select

Search: General keyword

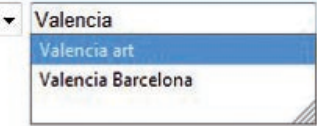

most popular keywords from the last 2 days...

accounting action business care children china christopher control criminal design e

food frank goldberg handbook health history information international management media midwifery model nursing oliver palmer pathophysiology schlesinger science social sport stafford systems technology women

El opac de la Univ. de Huddersfield es simple pero proporciona una gran cantidad de sugerencias y ayudas a la búsqueda

\section{Vídeos de promoción de bibliotecas premiados por} Information Today

http://www.infotodayblog.com/2007/04/17/the-first-infotubeyaward-ceremony/

1. "What's up at the library?"

Arlington Heights Memorial Library, Arlington Heights, IL http://www.youtube.com/watch? $v=B Z c w 9 Z x 4 e j A$

2. "I love the library"

McCraken County Public Library, Paducah, KY http://www.youtube.com/watch? $v=$ 3z7VGJSrQ4\&NR = 1

3. "Three reasons why I love my library"

KYNew Jersey State Library, Trenton, NJ $h+t p: / / w w w . y o u t u b e . c o m / w a t c h ? v=Z e Q / 25 n 8 q P Q$

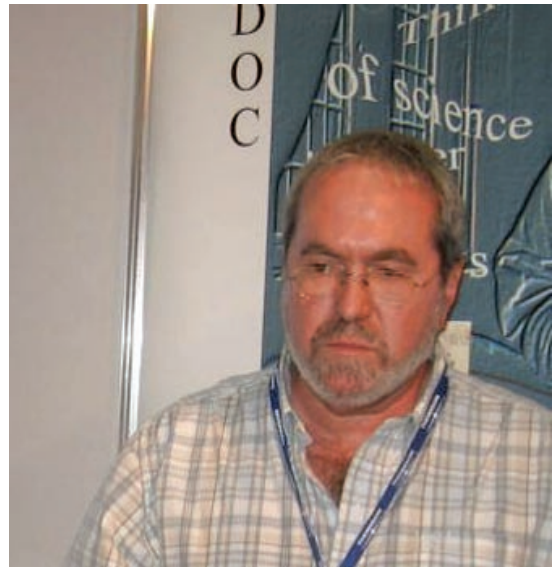

Lluís Codina, UPF, hizo una presentación sobre "Bancos de imagen y sonido en la Web" 


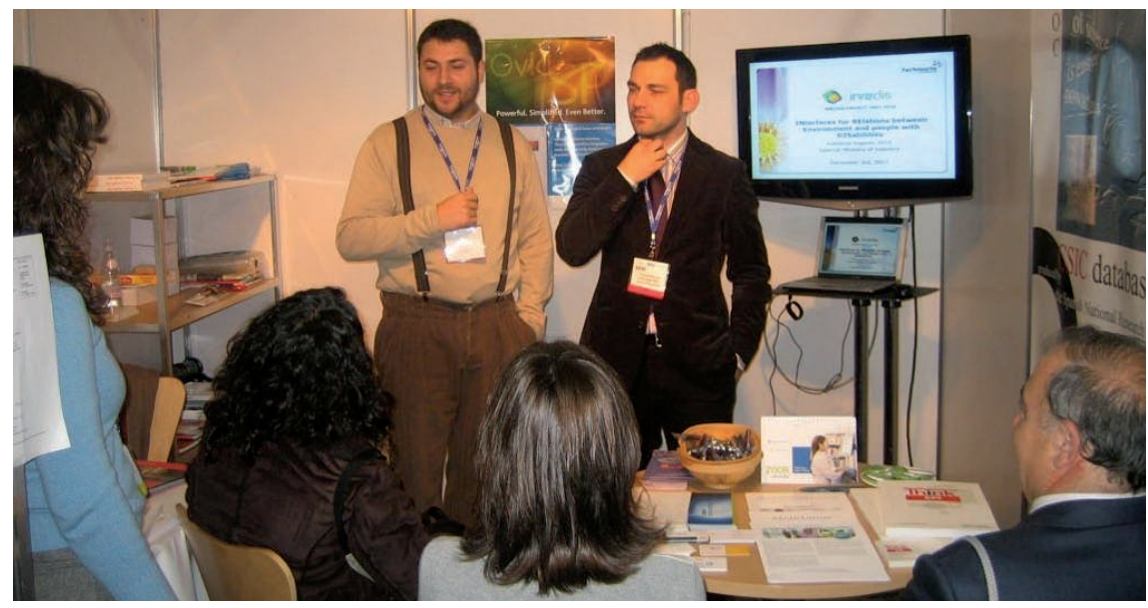

Comunicación de Pablo Lara, UOC, y José Á. Martínez-Usero, UCM, sobre "Research trends on disabled technologies for information services access in Spain"

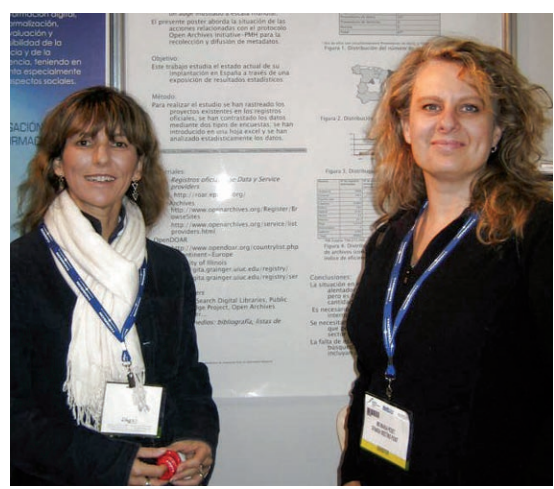

Antonia Ferrer y Fernanda Peset ante su poster "Implantación del protocolo OAI-PMH en España"

\section{Babyboomers ${ }^{1}$ versus generación MySpace}

Como hemos dicho, varias sesiones se dedicaron a analizar las expectativas y desafíos que plantea la nueva generación de estudiantes que ahora sale de la escuela. La opinión general es que ponen poca atención y que aprenden sobre todo jugando y experimentando. Un orador nos instó a que no sólo diseñemos los productos con ellos en mente, sino a ir más lejos: darles las riendas y dejar que sean ellos los que diseñen sus productos.

Siendo condescendientes con los oradores que exageraban las supuestas diferencias, en aras de que ello quizá hacía su ponencia más novedosa, siempre es bueno escuchar esas investigaciones sobre el comportamiento de los jóvenes tecnificados. La gente de $O C L C$ y de ProQuest han realizado varios estu- algún curso les había dado pautas de búsqueda, o los que recientemente habían asistido a un programa de extensión bibliotecaria. Los que intentaron el "juega, a ver qué pasa", o cayeron en la frustración creyendo que no había nada disponible, o encontraron sólo un par de cosas secundarias sin hallar el mejor material.

Jim Ashling, de Information Today, es otro de los que cree que no hay tanta diferencia entre las generaciones como algunos dicen, y remitió a los estudios de Oclc y ProQuest.

Estudio de Oclc (19 pp., 52 $\mathrm{KB})$ : dios de comunicación que eligen, su paciencia o falta de la misma con los sistemas poco amigables, su conocimiento de los recursos existentes, el nivel de confianza en ellos...

John Law, de ProQuest, informó que, en contraste con la sabiduría popular que da por hecho que Google es el único sitio donde buscan los estudiantes, la realidad demuestra que están mucho más sensibilizados y respetan la calidad de los recursos electrónicos convencionales suministrados por las bibliotecas. La mala noticia es que, si bien los estudiantes parecen saber que la biblioteca posee gran cantidad de 'materiales buenos', su alfabetización informativa es tan pobre que les cuesta mucho encontrarlos.

Contó que se habían analizado los resultados de cada uno de los estudiantes de un proyecto, y se contrastaron con la educación informacional que habían recibido anteriormente. Los que hicieron un mejor trabajo fueron los que en algún momento del pasado el tutor de

http://www.oclc.org/research/ publications/archive/2007/connawayacrl.pdf

Estudio de ProQuest:

http://www.proquest.com/ pressroom/pressrelease/07/20071105. shtml

Véase también la nota ThinkEPI de Jesús Tramullas:

http://www.thinkepi.net/nativosdigitales

Ian Rowlands, de la School of Library, Archival and Information Studies (Slais) del University College London, presentó un estudio que había hecho sobre dicha "generación". También corroboró que, contrariamente a la creencia popular, esos jóvenes no son necesariamente unos usuarios intuitivos de internet. Rowlands observó una continuidad entre las generaciones, y no una ruptura radical. Señaló que muchos de los llamados silver surfers (surfistas de plata, de más de 60 años) son, de hecho, más amantes de la
"Uno de los temas repetidos fue el comportamiento de los usuarios jóvenes, a los que se les llamó generación Google, generación MySpace, nativos digitales, screenagers, generación del Milenio y aún más" 
tecnología que los adolescentes, algunos de los cuales eran verdaderos "disidentes digitales" al elegir conscientemente no usar internet. También constató la visión simplista de los adolescentes ya que ven internet como Google y "todas las cosas contenidas" en Google. Su conclusión fue que había en general una sobre-estimación del efecto de internet en los jóvenes, y una subestimación de otros grupos demográficos.

Todo eso vino muy bien para corregir lo que a los evangelistas de la Web 2.0 les gusta tanto decir.

\section{Web 2.0 en la empresa}

En la sesión titulada "Web 2.0 en acción" se presentaron casos de implementación de redes sociales en empresas, incluido un blog en el que participaba el director general de la compañía. Poner en marcha una herramienta participativa es interesante por lo que representa de cambio de la cultura empresarial, ya que al permitir comentarios, disentir y hacer auto-exámenes críticos todo se democratiza. La Web 2.0 no es una "fuerza mística", sino que ofrece algunas soluciones prácticas para los problemas de la vida real, como la falta de tiempo y de dinero, dos problemas acuciantes para las personas que trabajan en el tercer sector.

Se produjo una situación divertida cuando una persona de Airbus preguntó cuál era la mejor manera de favorecer la adopción de las tecnologías web 2.0 en su organiza- ción, y el orador le aconsejó: “sólo déjeles jugar con ello, pues como más se aprende es con los fallos". La gente se rió cuando lo asoció también a posibles fallos del Airbus.

\section{Gestión del conocimiento (KM): comunidades}

La gestión de conocimiento también se ve invadida por la ola 2.0, y la gente que trabaja en ello dio cumplida cuenta de las nuevas facetas que va adquiriendo, por ejemplo usando blogs. Es lógico, porque todo lo 2.0 encaja como anillo al dedo a la KM, tratándose de que afloren y se compartan experiencias y saberes.

Lilia Efimova (Telematica Instituut, Holanda) dijo al respecto: "Los blogs corporativos proveen una oportunidad sencilla y fácil para que los empleados compartan libremente ideas sobre temas, no obligados por fechas límite sino abiertos al futuro de la organización. En el blog aparecen espontáneamente lecciones aprendidas, con nuevos matices que escapan a los informes oficiales. Asimismo está demostrado que en los blogs se producen brainstormings y serendipidad, y se generan inesperadas nuevas ideas".

La oradora ofreció unos consejos para poner en marcha un blog en una compañía:

- Dejar bien claro ante todos que el blog está apoyado por la dirección, así como explicitar los temas que no deben ser blogueados en público.
- Hablar con los mandos intermedios sobre los beneficios y los riesgos de un blog, con el fin de que le pierdan el miedo.

- Dar cursillos y buscar el apoyo de empleados que ya conozcan los blogs. Atender y cuidar a los nuevos participantes.

- Tratar también temas "antiguos". Algunas sugerencias: informes de visitas y viajes, apuntes de cursos, situación de proyectos, comentarios sobre productos de la competencia...

- Hacer que bloguear sea algo usual, integrándolo en otras actividades, e incorporando su información en otros informes y documentos institucionales.

Por su parte, la comunicación del consultor David Gurteen trató de "La aparición de la KM social" y comentó algunos de los 31 vídeos breves (de 1 a 2 min de duración) de entrevistas, muy interesantes, que él ha publicado en YouTube:

http://www.youtube.com/results? search_query $=$ Gurteen + miniinterview\&search $=$ Search

En ellos Gurteen pregunta a directivos cómo ven la KM y las herramientas sociales.

Alguien nos recomendó un grupo de administradores de intranets corporativas que se llama Intranetters. Tienen una lista de correo bastante dinámica (unos 35 mensajes mensuales) en la que los miembros discuten y responden a las preguntas de los demás directores de intranet.
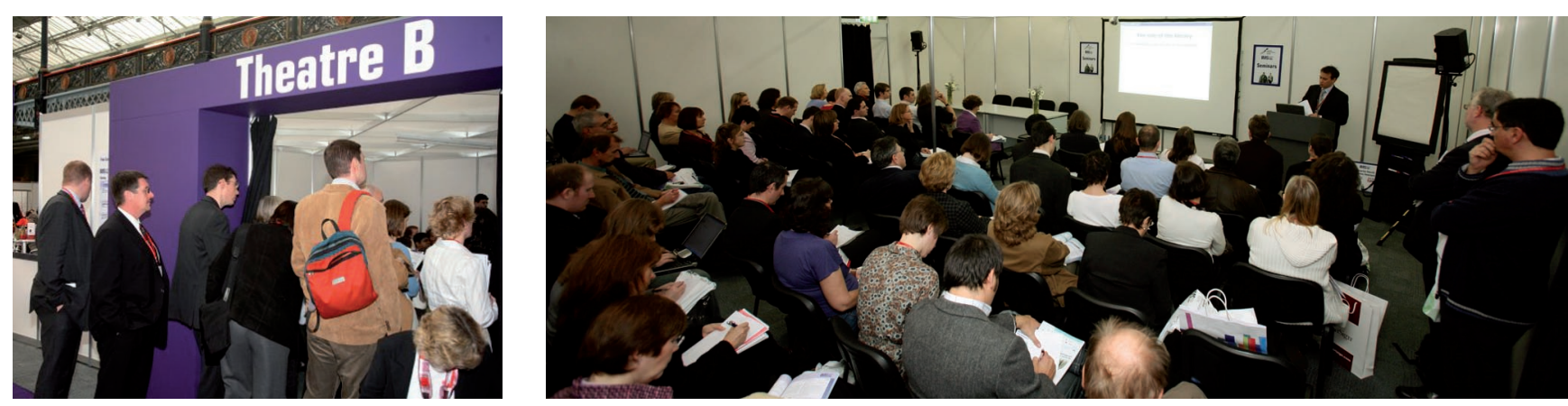

Seminario en un "theatre" de la zona de la feria. Son interesantes y gratuitos, por lo que tuvieron gran éxito de público 


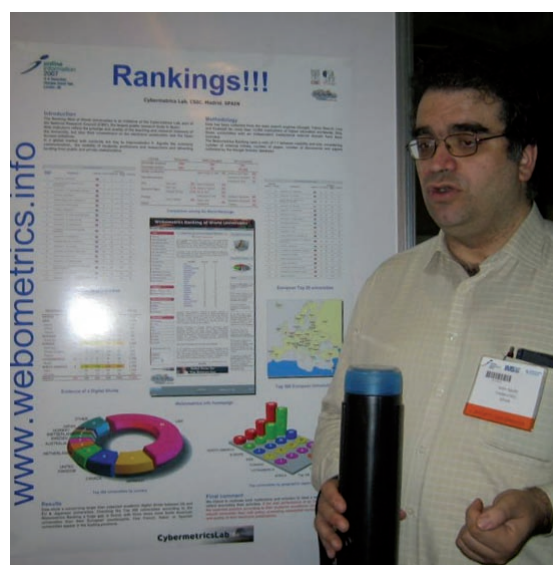

Isidro Aguillo, ledcyt/CSIC. El rótulo de su poster "Rankings of World universities" atrajo muchas visitas

http://tech.groups.yahoo.com/ group/intranetters/

Empezó en Londres en junio de 2007 , pero ya tiene algunos miembros en el continente.

\section{Futuro de la web colaborativa: 3.0 y más allá}

En la mesa redonda se discutió sobre la privacidad, así como sobre el peligro de la falta de honestidad y de movimientos tendenciosos y sesgados en la Red. Richard Dennison $(B T)$ defendió la idea de estándares abiertos y la transparencia como un antídoto (aunque no una cura para todos) para atajar la parcialidad y el eventual uso de la web 2.0 por parte de mafias. En esencia, su argumento se basa en la cita: "Eterna vigilancia es el precio de la libertad" (Eternal vigilance is the price of liberty. Los indolentes verán como sus derechos se convierten en presa de los activos -John Philpot Curran, 1790), pero esto plantea la cuestión de quiénes son los que nos proporcionan la vigilancia.

\section{Los workshops de la feria}

No todas las sesiones se producen en la parte de conferencias de la Online Information. Abajo en la feria (con entrada libre) tienen lugar seminarios concurrentes de media hora de duración, a los que cualquier visitante puede acceder. Uno bastante interesante fue el de Kate Worlock, de Outsell, sobre "desarrollo ágil". Caracterizó el clima actual de la publicación como uno en el que los clientes 'controlan' mucho más la producción indicando sus preferencias, desean experimentar como cualquier otro consumidor, y tienen déficit de atención. Los competidores aligeran sus cargas fijas de producción para poder acometer cambios rápidos a voluntad, sobre la marcha. Después de describir el "círculo virtuoso" iterativo e interactivo de la publicación ágil: describir, desarrollar, probar, lanzar, aprender, y empezar de nuevo, preguntó a los asistentes si pensaban que sus procesos eran ágiles. "Debéis pensar en tener plataformas y marcos flexibles en lugar de los efímeros productos", fue su consejo. La realimentación (feedback) es fundamental en el desarrollo ágil, ya que permite ir mejorando los productos sobre la marcha. Por ello considera que el lanzamiento de un producto es el comienzo, no el final.

\section{Spanish Meeting Point}

A propuesta de esta revista, la ya tradicional Sesión Española que se celebraba en la Online Information Conference desde 1989, se convirtió este año en el Spanish Meeting Point, un stand durante los 3 días $^{2}$. Aunque el stand tuvo que estar atendido por personal y colaboradores de EPI, se demostró su utilidad:

- como plataforma de maniobra de empresas españolas en la Conferencia, a las que no compensa pagar un stand completo, y sobre todo tener personal allí fijo; con esta solución los representantes de las organizaciones pueden moverse por la feria y concertar entrevistas, o hacer presentaciones de sus productos usando el stand según un horario establecido;

- para que varios investigadores españoles pudieran presentar ponencias y posters (en total fueron 6);

- como punto de encuentro para los españoles visitantes; en relación con esto tuvimos la sorpresa de conocer a una docena de españoles que están trabajando en el Reino Unido como bibliotecarios y documentalistas.

El stand fue ofrecido gratuitamente por los organizadores, y esta revista EPI sufragó parte del mobiliario y el alquiler de un PC y una pantalla LCD. Las empresas españolas participantes sólo tuvieron que abonar una cantidad para disponer de catering en el stand, algo que los visitantes de la feria siempre agradecen.

Este año 2008 volveremos a tener Spanish Meeting Point. Allí os esperamos.

\section{Notas}

1. El término baby boomer se aplica en los países de habla inglesa a las personas nacidas entre 1946 y 1962 (los que ahora en 2008 tienen entre 46 y 62 años). Después de la $2^{\text {a }}$ Guerra mundial se dió un boom de nacimientos en los países vencedores. En España el boom llegó unos 14 años más tarde, por lo que los baby boomers españoles tienen hoy 32-48 años aproximadamente.

2. Hace años la Asociación Española de Distribuidores de Información Electrónica (Asedie) ya había instalado un stand colectivo para todos sus miembros en la feria.

3. Ruiz-Pérez, Rafael; Jiménez-Contreras, Evaristo; Delgado-López-Cózar, Emilio "Complementos bibliométricos de Thomson Scientific en la Web: buenos, bonitos y gratuitos". En: El profesional de la información, 2008, v. 17 , n. 5 , septiembre-octubre, pp. 559-563.

4. Este informe se ha complementado con algunos comentarios tomados de estas webs: http://www.online-information.co.uk/ http://annewelsh.wordpress.com/ http://neilstewart.wordpress.com http://infolitweb.blogspot.com/ http://blog.360.yahoo.com/ http://dawn-onlineinformation2007.blogspot.com/ http://netjmc.typepad.com/

http://www.infotodayblog.com/2007/12/

Tomàs Baiget, Institut d'Estadística de Catalunya (Idescat)

baiget@sarenet.es

Fernanda Peset, Universidad Politécnica de Valencia (UPV)

mpesetm@upv.es 